\title{
IT-Sektor: Chancen für die deutsch-nigerianische Wirtschafts- und Entwicklungspartnerschaft
}

\author{
Die Entstehung und das Wachstum des größten afrikanischen Markts für Informations- \\ und Kommunikationstechnologie (IKT) in Lagos beruhen auf Agglomerationseffekten und \\ dem Zusammenspiel komplementärer Erfolgsfaktoren, die mit positiven externen Effekten \\ behaftet sind. Notwendige Reformen, die sowohl durch nationale Maßnahmen zur Beseitigung \\ von Marktversagen als auch durch internationale Maßnahmen der Entwicklungsarbeit \\ implementiert werden sollen, erfordern eine Überführung des Marktes in den formellen \\ Sektor. Dabei besteht die Gefahr, das Zusammenspiel der Erfolgsfaktoren zu zerstören. \\ Die Chancen und Risiken dieser Aspekte für die deutsch-nigerianische Wirtschafts- und \\ Entwicklungszusammenarbeit werden aufgezeigt.
}

Es ist wichtig, wirtschaftliche Wachstumspotenziale in $\mathrm{Ni}-$ geria und anderen Partnerländern gezielt zu nutzen, um durch die Verbesserung der wirtschaftlichen Lage die dortigen Lebensbedingungen zu verbessern und die Ursachen für Flucht und weitere Formen der zwangsweisen Migration zu bekämpfen. Die Zuwanderung vom afrikanischen Kontinent erfolgt in erster Linie aus Nigeria, Somalia und Eritrea. Gerade Nigerianer:innen stellten mit einem Anteil von 6,4 \% 2019 und 3,1\% 2020 an der Gesamtzahl der in Deutschland Asylsuchenden eine nicht zu vernachlässigende Asylbewerbergruppe dar. Jedoch wurden 2020 52,7\% der von Nigerianer:innen gestellten Asylanträge abgelehnt. In weiteren $39,1 \%$ der gestellten Anträge ergingen formelle Entscheidungen, dass z. B. ein anderer Mitgliedstaat zuständig sei, das Verfahren aufgrund der Antragsrücknahme der Antragstellenden eingestellt werde oder im Folgeantragsverfahren entschieden wurde, kein weiteres Asylverfahren durchzuführen (BAMF, 2021, 7, 10, 17, 35, 42).

Vor dem Hintergrund dieser niedrigen Bewilligungsraten kommt dem Abbau von Fluchtursachen und der Reintegration der Rückkehrenden nach Nigeria eine wichtige Rolle zu. Das Programm „Perspektive Heimat“ des Bundesministeriums für wirtschaftliche Zusammenarbeit und Entwicklung (BMZ) möchte daher aus Deutschland in ihre Heimatländer zurückkehrenden Personen Jobangebote in diesen Ländern machen (BMZ, 2021a). Somit stellt die Förderung der wirtschaftlichen Entwicklung in den Heimatländern der Asylsuchenden einen zentralen Aspekt der deutschen Wirtschafts- und Entwicklungszusammenarbeit dar.

(C) Der/die Autor:in 2021. Open Access: Dieser Artikel wird unter der Creative Commons Namensnennung 4.0 International Lizenz veröffentlicht (creativecommons.org/licenses/by/4.0/deed.de).

Open Access wird durch die ZBW - Leibniz-Informationszentrum Wirtschaft gefördert.

\section{Die Lage in Nigeria}

Nigeria verfügt über wirtschaftliches Potenzial. So ist Nigeria in Subsahara-Afrika Deutschlands zweitwichtigster Handelspartner mit Exporten in Höhe von 891,6 Mio. Euro und Importen in Höhe von 1.596,6 Mio. Euro im Jahr 2020 (Statistisches Bundesamt, 2021). Das Land ist 2020 mit etwas über 206 Mio. Einwohner:innen das bevölkerungsreichste Land Afrikas (United Nations, 2019a, 25). Zudem ist die Bevölkerung überdurchschnittlich jung: Das Durchschnittsalter lag 2020 bei nur 18,1 Jahren (United Nations, 2019b) - viele potenzielle Arbeitskräfte, „ein enormes kreatives Potenzial“, wie auch der Bundesminister für wirtschaftliche Zusammenarbeit und Entwicklung, Gerd Müller, feststellte (Becker und Goffart, 2021). Doch gerade mit Blick auf eine Arbeitslosenquote der 15- bis 34-Jährigen von 42,5\% (National Bureau of Statistics, 2021a) und darauf, dass 39,1\% der Bevölkerung 2018 unter der Armutsgrenze von 1,90 US-\$ pro Tag und Person lebte (Weltbank, 2021), bedarf es noch einiger Anstrengungen, dieses Potenzial zu heben.

Doch dazu fehlt es unter anderem an Bildungsangeboten. Die Einschulungsrate betrug 2016 knapp 85\% (Unesco,

PD Dr. Johannes Paha ist Privatdozent an der Justus-Liebig-Universität in Gießen, wo er zur Wettbewerbs- und Entwicklungspolitik forscht.

Lydia Wolter studiert Volkswirtschaftslehre, Anglistik und Amerikanistik an der Universität Potsdam. 
O. J.). Obwohl die Grundschulbildung von 6 bis 14 Jahren kostenlos und verpflichtend ist, besuchen nur $61 \%$ der 6 bis 11-Jährigen regelmäßig die Schule (Unicef, o.J.). Die Alphabetisierungsrate der über 15-Jährigen liegt daher nur bei knapp $60 \%$ (Unesco, o.J.). Die Lernbedingungen an den Schulen sind angesichts des niedrigen staatlichen Budgets mangelhaft (Ulrich, 2017). 2018 wurden nur $7 \%$ der Staatsausgaben für Bildung ausgegeben. Die Empfehlung der Unesco liegt jedoch bei $15 \%$ bis $25 \%$ (Ekundayo, 2019). Auch die Qualität der Hochschulbildung hat dringenden Verbesserungsbedarf, um dem Fachkräftemangel entgegenzuwirken. Hohe Gebühren von privaten Universitäten stellen eine große Hürde dar. An öffentlichen Universitäten kommt es aufgrund der niedrigen Staatsausgaben im Bildungssektor regelmäßig zu Streiks (Ekundayo, 2019). Stattdessen erfolgen Berufsausbildungen mitunter selbstorganisiert durch Learning by Doing im informellen Sektor. Gerade der IKTSektor bietet laut einer Analyse der Wirtschaftsprüfungsgesellschaft PwC (2016) gute Bedingungen, um Wissensaneignung und -weitergabe on the job zu fördern und so die gesamtwirtschaftliche Produktivität zu steigern.

Trotz dieser schwierigen Rahmenbedingungen nimmt Nigerias wirtschaftlicher Erfolg stetig zu. Das nigerianische Bruttoinlandsprodukt (BIP) überholte das südafrikanische bereits 2014, wenngleich das BIP pro Kopf noch immer um mehr als den Faktor 2,5 auseinanderfällt (Weltbank, 2020). Dennoch macht dies Nigeria zur größten Volkswirtschaft Afrikas, hauptsächlich aufgrund der Erdöl- und Erdgasvorkommen. Doch dieser Erfolg hat seinen Preis. Die wirtschaftliche Abhängigkeit von Rohstoffen ist kritisch zu beurteilen, auch angesichts der weltweiten Bemühungen zum Klimaschutz. Wichtig ist mit Blick auf die wirtschaftliche Entwicklung Nigerias, eine Diversifizierung zu erreichen, um die starke Abhängigkeit von Erdgas und -öl aufzubrechen, die zwar entscheidend zum Wirtschaftswachstum beiträgt, aber nur wenige Arbeitsplätze schafft (GTAI, 2020). Gerade im Bereich IKT ist Lagos, Nigerias größte Stadt und die zweitgrößte des Kontinents, aktuell vielversprechend aufgestellt. Hierzu gehört das Otigba Computer Village.

\section{Maßnahmen der Entwicklungszusammenarbeit}

Das Spannungsverhältnis zwischen Chancen und Herausforderungen innerhalb des Landes betrifft auch Maßnahmen der wirtschafts- und entwicklungspolitischen Zusammenarbeit, deren Wirksamkeit durch diese Rahmenbedingungen herabgesetzt wird. Ein Beispiel für solche Maßnahmen ist das BMZ-Programm „Perspektive Heimat“. Dieses möchte aus Deutschland in ihre Heimatländer zurückkehrenden Menschen den beruflichen Neustart erleichtern. Schließlich finden die Rückkehrenden dort oft nur wenige formalisierte Arbeitsverhältnisse vor und sind von häufigen Jobwechseln betroffen. Das Pro- gramm betreibt lokale Beratungszentren und möchte den Rückkehrenden aus Deutschland ein Jobangebot im Herkunftsland machen, um so Bleibe- und Zukunftsperspektiven zu schaffen. Ein Baustein sind auch finanzielle Zuschüsse für Unternehmensgründungen. Das Programm wird durch eine Reihe von Projekten z. B. von der Deutschen Gesellschaft für Internationale Zusammenarbeit (GIZ) umgesetzt (BMZ, 2021a).

Die Umsetzung dieser Angebote durch Organisationen wie die GIZ erfordert, auch mit Blick auf die Rechenschaftspflicht gegenüber dem BMZ als Auftraggeber und den politischen Trägern im Partnerland, formalisierte Strukturen und Arbeitsverhältnisse. Gleichzeitig kann diese Formalisierung den Projekterfolg gefährden, da der durch die Formalisierung bewirkte Kostenanstieg für Löhne und Sozialversicherungsabgaben zum Arbeitsplatzabbau beitragen kann. Eine Akzeptanz informeller Beschäftigungsverhältnisse dürfte zwar keine Alternative sein. Jedoch sollte die gezielte fachliche (möglicherweise aber auch finanzielle) Unterstützung bei der Formalisierung von Arbeitsverhältnissen Teil der Programme sein.

Davon sind auch die Digitalisierungsaktivitäten des BMZ inklusive seiner Initiative „Digitales Afrika“ betroffen, zu der die „Strategische Partnerschaft Technologie in Afrika“ in Kooperation mit deutschen Unternehmen gehört. Hierbei geht es sowohl um den Einsatz digitaler Technologien in Entwicklungsprojekten als auch um die Förderung öffentlichprivater Kooperationsprojekte in afrikanischen Ländern, um durch den Einsatz digitaler Technologien zur Erreichung der globalen Nachhaltigkeitsziele beizutragen (BMZ, 2021b).

\section{Erfolgsfaktoren der Entwicklung im IKT-Sektor}

Nigerias IKT-Sektor machte im ersten Quartal 2021 9,91\% des nominalen BIP aus und zählt somit zu den wirtschaftlich relevantesten Sektoren des Landes (National Bureau of Statistics, 2021b): Er gilt als einer der Sektoren, dessen Leistung 2020 dazu beigetragen hat, das Land aus der Rezession zu führen (Nigerian Communications Commission, 2021). ${ }^{1}$ Zudem identifiziert z. B. PwC (2016) IKT als einen der Sektoren mit den stärksten positiven Auswirkungen auf die nigerianische Gesamtwirtschaft. Angesichts der notwendigen Diversifizierung der rohstoffabhängigen Wirtschaft ist dieses Potenzial entscheidend, wenn es darum geht, von bisher ungenutzten Wachstumschancen zu profitieren und andere Sektoren mithilfe von IKT voranzutreiben. Das erkannte die nigerianische Regierung auch in ihrem „Economic Recovery and Growth Plan“ (Februar 2017) an, in dem der Markt als wichtiger Wegbereiter für

1 Eine gekürzte Darstellung dieser Erfolgsfaktoren findet sich auch bei Paha und Wolter (2021). 
internationale Wettbewerbsfähigkeit erachtet wird, sowie in ihrer 2019 veröffentlichen „National Digital Economy Policy and Strategy“ (2020-2030), die darauf abzielt die nigerianische Wirtschaft vor allem im digitalen Bereich neu aufzustellen, um die Abhängigkeit von Öl und Gas zu reduzieren (International Trade Administration, 2020). Der Aufwärtstrend der vergangenen Jahre bleibt international nicht unbemerkt: So bestätigte Facebook-Gründer Mark Zuckerberg 2016 bei einem Besuch in Lagos die aussichtsreiche Zukunft der dortigen Internet- und Social-MediaBranche. Auch Google hat den Trend erkannt und 2018 ein „Accelerator Program“ initiiert, um Unternehmensgründungen zu unterstützen (Scherer, 2018). Einer der bekanntesten Nachwuchspolitiker Nigerias, Uche Chuta, ist überzeugt: „Die Tech-Industrie ist die beste Chance, die das Land hat" (Scherer, 2018). Das starke Wachstum dieser Industrie von unter $1 \%$ auf etwa $10 \%$ Anteil am BIP (OC\&C Consulting, 2018) bietet der wachsenden, technikaffinen Bevölkerung Beschäftigungsmöglichkeiten und eine Perspektive für langfristig verbesserte Lebensbedingungen.

Ein populäres Beispiel für das Potenzial des IKT-Sektors ist „Westafrikas Silicon Valley“ - wie das Otigba Computer Village in Lagos mitunter bezeichnet wird (Oyeyinka, 2017). Otigba dient als Marktplatz für den Verkauf von IKTProdukten und für damit verknüpfte Dienstleistungen, wie z. B. die Reparatur gebrauchter Hardware. Folglich unterscheidet sich Otigba zwar vom US-amerikanischen Silicon Valley als Innovationsstandort, doch ist Otigba mehr als ein Marktplatz unter vielen: Mit über 3.000 (meist kleinen und mittleren) Unternehmen und einem Tagesumsatz von rund 1,5 Mrd. nigerianischen Naira (ca. 3 Mio. Euro; Success Nwogu, 2018) wird Otigba häufig als Afrikas größter IKT-Markt bezeichnet (Oyelaran-Oyeyinka, 2006).

Zu den Schlüsselfaktoren für Otigbas Entwicklung gehören positive externe Effekte der Bildung. So verfügt dort ein für nigerianische Verhältnisse überdurchschnittlich hoher Arbeitskräfteanteil über eine Hochschulausbildung (ca. $50 \%$ ) oder eine technische Ausbildung (ca. $30 \%$; Oyeyinka, 2017). Dies trägt entscheidend zum Erfolg des Clusters bei, da diese Fachkräfte ihr Wissen und ihre Fertigkeiten an andere Beschäftigte weitergeben (Jegede, 2019). Z.B. werden durch Training on the Job junge Menschen in vielfältige Tätigkeiten wie Batteriereparaturen, den Handel mit gebrauchten Handys oder den Verkauf von Software eingewiesen. Diese Form der Ausbildung ist angesichts der eingeschränkten Bildungsmöglichkeiten in Nigeria besonders relevant, da sie jungen Menschen eine Chance gibt, auf eigenen Beinen zu stehen. Dabei werden die Auszubildenden selbst zu Experten und haben die Möglichkeit, verantwortungsvollere Tätigkeiten zu übernehmen oder ihr eigenes Geschäft zu eröffnen. Sie fungieren als Multiplikator:innen zur Weitergabe der erlernten Fertigkeiten (Salaudeen, 2018).
Einen weiteren Schlüsselfaktor stellt die hohe Konzentration der wirtschaftlichen Aktivität auf engem Raum dar. Viele der Unternehmen in Otigba sind klein und profitieren von der aggregierten Größe des Clusters (Abiola, 2008). So sind Unternehmen innerhalb des Clusters produktiver als Unternehmen außerhalb des Clusters (Ogunjemilua et al., 2020). Denn durch die Anhäufung vieler Handelnder der gleichen Branche an einem Ort kann sich Otigba Agglomerationseffekte in Form von externen Skalenerträgen zunutze machen. Unternehmen können also von sinkenden Kosten aufgrund der aggregierten Größe des Clusters profitieren (Porter, 1998). Beispielhaft ist hier die Reduktion der Beschaffungskosten von Inputgütern aufgrund des Vorhandenseins und der Inanspruchnahme der Leistungen spezialisierter Importeure oder Zwischenhändler zu nennen.

Auch Wissens-Spillovers sind ein Beispiel für erstrebenswerte Agglomerationseffekte (Carlino und Kerr, 2015). Dabei handelt es sich um den beabsichtigten oder unbeabsichtigten, oft unternehmensübergreifenden Austausch von für die jeweilige Tätigkeit relevantem Wissen durch z.B. informelle Gespräche oder Mitarbeiterwechsel. Solche Wissensübertragungen sind in Otigba unter anderem durch die innerbetriebliche Ausbildung, durch die hohe Beschäftigungsmobilität der Arbeitnehmenden, die zwischen verschiedenen Unternehmen wechseln, sowie durch die hohe Zahl an Start-ups zu beobachten (Ogunjemilua et al., 2020). Hier offenbart sich auch der Village-Charakter Otigbas als weiterer Schlüsselfaktor: Das Computer Village ist für eine kollaborationsfreudige Gemeinschaft bekannt, in der die Mitglieder eng miteinander verbunden sind und sowohl Ressourcen als auch ihr Wissen miteinander teilen. So trägt dieser Wissensaustausch zwischen Unternehmen dazu bei, dass Unternehmen ihre Geschäfte mitunter bereits innerhalb der ersten drei Jahre ihrer Tätigkeit ausweiten und ihr Eigenkapital steigern können (Jegede, 2019).

Ein weiteres Beispiel für die Nutzung externer Skalenerträge zeigt sich am Arbeitsmarkt. So steigert die hohe Konzentration an ähnlich qualifizierten und spezialisierten Arbeitskräften sowohl die Quantität als auch die Qualität an erfolgreichen Matches zwischen Arbeitgeber:innen und Arbeitnehmenden. Folglich steigt die Produktivität durch eine bessere Passung der angebotenen und nachgefragten Qualifikationen. Ebenso wird Unsicherheit auf beiden Seiten reduziert, denn Unternehmen können besser auf unvorhersehbare Ereignisse und Herausforderungen reagieren, indem sie sich die Verfügbarkeit von qualifizierten Arbeitskräften zunutze machen. Gleichzeitig können hoch spezialisierte Arbeitskräfte schnell und unkompliziert von einem Unternehmen zum anderen wechseln, ohne lange Fahrtwege oder einen Umzug in Kauf nehmen zu müssen (Carlino und Kerr, 2015). 
Ein weiterer Schlüsselfaktor, der das kleine Computer Village auf seine heutige Größe anwachsen ließ, war die Nutzung von First-Mover-Vorteilen. Otigba war der erste Marktplatz, der parallel zum Verkauf von IKT-Produkten eine ergänzende Dienstleistungsindustrie für die Produkte entwickelte (Asemota, 2017). So können Kund:innen gleich den passenden Mobilfunkvertrag zum neuen Handy abschließen, bei einem Laptopkauf die Software installieren lassen oder weiteres Zubehör erwerben. Dass dieses Angebot angenommen wurde, lag entscheidend an einer Erhöhung der Markttransparenz durch Werbung in den lokalen und überregionalen Medien. Da Produktpreise in Zeitungen veröffentlicht wurden, konnten Abnehmer:innen jederzeit die günstigste Quelle für ihre Produkte finden. So kamen schließlich Kund:innen aus ganz Nigeria und darüber hinaus nach Otigba, um die dort angebotenen Produkte und Dienstleistungen zu erwerben (Asemota, 2017).

\section{Marktversagen, staatliche Eingriffe und Entwicklungszusammenarbeit}

Das Wachstum des Clusters hat jedoch auch nachteilige Folgen. So kam es zu Marktversagen in Form von negativen externen Effekten, die für schnell und auf begrenztem Raum wachsende Cluster nicht untypisch sind (Sedgley und Elmslie, 2004; Richardson, 1995). Schließlich war das Otigba Computer Village ursprünglich als reine Wohngegend vorgesehen. Das exponentielle Wachstum des Clusters überstieg die verfügbaren Kapazitäten. So wurden Umweltbelastungen wie z. B. Krankheiten und Ungezieferplagen durch nicht abtransportierte Abfälle zu einem großen Problem. Die Abwassernetze sind von Müll verstopft, weshalb eine erhöhte Überschwemmungsgefahr herrscht. Und auch Staus aufgrund der überlasteten Infrastruktur (z. B. durch eingestürzte Brücken), Wohnungsmangel und hohe Mieten bzw. Immobilienpreise aufgrund des begrenzten Platzes behindern die erfolgreiche Weiterentwicklung des Clusters (Chen, 2019).

Die Reduktion dieser Formen des Marktversagens erfordert staatliche Eingriffe zur Internalisierung der negativen externen Effekte. Den Unternehmer:innen der TechBranche mangelt es an öffentlichen Gütern in Form einer regionalen Infrastruktur, z. B. Straßennetz, Parkplätze und ein zuverlässiger öffentlicher Nahverkehr. Der Zugang zu Kapital muss erleichtert werden. Zudem muss die ständige Benzin- und Dieselknappheit gelöst werden (GIZ, 2017). Darüber hinaus werden eine stabile Strom- und Internetversorgung, eine moderne Energieerzeugung (Päffgen, 2021) sowie eine zuverlässige Abwasserentsorgung benötigt (International Trade Administration, 2020). Auch der Abbau von Informationsasymmetrien und Faktoren, die Ungewissheiten und Risiken hervorrufen, wäre für die weitere Entwicklung Otigbas wünschenswert. Hierzu gehören rechtliche und regulatorische Maßnahmen zum Ab- bau von Handelshemmnissen, da die Komponenten und Produkte, die im Cluster angeboten werden, zu mehr als zwei Dritteln aus importierten Waren bestehen. Die Leistungsfähigkeit der Unternehmen im Cluster hängt also unmittelbar davon ab, welche Kosten den Handelnden für Importe entstehen. Des Weiteren treffen viele der politischen Richtlinien und institutionellen Hindernisse gerade kleine Unternehmen überproportional hart, wodurch das Wachstum und die Innovationsfähigkeit zusätzlich eingeschränkt werden (Abiola, 2008).

Der nigerianische Staat möchte diese Probleme durch eine Umsiedlung des Computer Village von Otigba nach Katangowa beheben. Aktuell werden dort noch die erforderlichen Baumaßnahmen durchgeführt, sodass die Umsiedlung ab ca. 2023 geplant ist (Vanguard News Nigeria, 2021). Während das Computer Village aktuell unter einer Übernutzung der vorhandenen Fläche leidet, soll der neue Standort auf über 15 Hektar genug Platz für die (staatlichen) Expansionspläne bieten (Technology Times, 2014). Gemäß den Planungen wird das neue IKT- und Innovationszentrum in drei Marktplätzen jeweils mehr als 1.000 Geschäfte beherbergen (Awosanya, 2018). Zudem ist die Bereitstellung von Parkplätzen, Be- und Entlademöglichkeiten sowie Lagerhallen und Raum für Montagetätigkeiten geplant. Auch Elektrizität soll zuverlässig verfügbar sein (Vanguard News Nigeria, 2020a) - ein entscheidender Vorteil in einem Land, in dem $40 \%$ der Bevölkerung gar keine Stromversorgung und mehr als die Hälfte der Bevölkerung keinen Zugang zu einer unterbrechungsfreien Stromversorgung hat (GIZ, 2020). Der nigerianische Staat verspricht sich durch die Umsiedlung und die so verbesserten Rahmenbedingungen eine weitere Beschleunigung des Wachstums des Computer Village und des nigerianischen BIP; zudem sollen zahlreiche neue Arbeitsplätze geschaffen werden (Onuoha, 2021). Auch das Investitionsklima kann so verbessert werden. Denn das Computer Village blieb bisher trotz des weltweit ungebrochenen Interesses an Investitionen in der nigerianischen Tech-Branche bei Investierenden eher unbeachtet. Ein Grund ist, dass es sich bei den hier ausgeübten Tätigkeiten hauptsächlich um ungeregelte wirtschaftliche Aktivitäten im informellen Sektor handelt, die den Investierenden weder die notwendigen Informationen zu einzelnen Unternehmen und dem weiteren Markt bieten können noch die notwendige Sicherheit bezüglich des Schutzes von Investierenden gegenüber betrügerischen Geschäftspraktiken (Salaudeen, 2018).

Zudem halten sich gerade internationale Kapitalgebende bei Informationsasymmetrien mit inren Investitionen deutlich zurück. Zwar sind Kreditmöglichkeiten innerhalb des Clusters vorhanden, jedoch sind die Kreditzinsen mit $27 \%$ bis $30 \%$ sehr hoch (Oyeyinka, 2017). Die Start-ups in Otigba würden daher von einem vereinfachten Zugang zu Finanz- 
dienstleistungen und Risikokapital profitieren. Entsprechende Anreize für Investierende könnte der nigerianische Staat z.B. durch Steuererleichterungen bei Kapitalgewinnen in der Frühphase der Investition schaffen, in der asymmetrische Informationen ein entscheidendes Investitionshindernis darstellen (International Trade Administration, 2020).

Ein Spannungsfeld zwischen den Erfolgsfaktoren für Otigbas bisherige Entwicklung, zu denen auch die in der Informalität begründete Flexibilität gehört, und Otigbas künftigen Entwicklungschancen tut sich auch bei der Beschäftigung auf. Die guten Beschäftigungsmöglichkeiten sind nicht uneingeschränkt positiv zu bewerten. Schließlich handelt es sich überwiegend um Beschäftigung im informellen Sektor (Jegede, 2019). Gäbe es genügend Arbeitsstellen im formellen Sektor, in dem das Arbeitsund das Sozialrecht sowie die nigerianischen Vorschriften zum Mindestlohn einzuhalten sind (Udoma und BeloOsagie, 2019), würden dem Wachstum unregulierter Tätigkeiten im informellen Sektor Grenzen gesetzt, und gut ausgebildete Fachkräfte wären nicht auf eine Beschäftigung im informellen Sektor angewiesen.

Allerdings kann eine solche Formalisierung auch negative Folgewirkungen haben. Denn wenn dadurch die Arbeitskosten erhöht werden, können bestehende Beschäftigungsverhältnisse beendet werden. Während diesem Arbeitsplatzverlust im Idealfall jedoch eine positive Verteilungswirkung z.B. aufgrund der Einhaltung der Mindestlöhne gegenübersteht, sind übermäßig bürokratische Prozesse und exzessive staatliche Eingriffe negativ zu beurteilen. Aktuell gelten langwierige Verzögerungen bei der Bearbeitung von Genehmigungen, Mehrfachbesteuerungen auf Bundes-, Landes- und Kommunalebene sowie die Zuständigkeit mehrerer Aufsichtsbehörden als die größten bürokratischen Herausforderungen (International Trade Administration, 2020). Die negativen Wirkungen der Formalisierung von Beschäftigungsverhältnissen stellen auch die Entwicklungszusammenarbeit vor Herausforderungen. Denn das Fehlen von Bildungs- und Beschäftigungsmöglichkeiten sowie von langfristigen Perspektiven begünstigt Arbeitslosigkeit und letztlich auch Migration. Daher ist es wichtig, negative Arbeitsmarkteffekte bei einer Formalisierung von Arbeitsverhältnissen bestmöglich zu vermeiden. Jedoch sind formelle Strukturen als Ansatzpunkte für die Entwicklungszusammenarbeit von grundlegender Bedeutung, damit diese Maßnahmen überhaupt erst ihre Wirkung entfalten können.

Ähnlich ambivalent ist die Umsiedlung Otigbas zu beurteilen, die mit der Überführung der Tätigkeiten in den formellen Sektor Hand in Hand geht. Denn durch die Umsiedlung könnten die Agglomerationseffekte sowie die positiven externen Effekte der Bildung zerstört werden. Eine der großen Sorgen ist, dass aufgrund der dezent- ralen Lage die Kund:innen ausbleiben (Omolayo, 2017). Zudem könnte sich die Notwendigkeit zum Erwerb eines Geschäfts als Markteintrittsbarriere oder als Hindernis für die Umsiedlung erweisen, da Handelnde trotz beabsichtigter Finanzierungserleichterungen (z.B. teilweiser Verzicht auf Sicherheiten) die für den Erwerb der Ladenflächen nötigen Mittel womöglich nicht aufbringen können (Vanguard News Nigeria, 2020b). Hinzu kommt gesellschaftliche Kritik, da der neue Standort vor der Bebauung kein ungenutztes Land war. Dort befand sich bereits ein Kleidungsmarkt, der unter ähnlichen Problemen wie das Computer Village litt, aber für die Umsiedlung des Computer Village beseitigt wurde (Famuyiwa, 2020).

\section{Diskussion}

Das Beispiel Otigba Computer Village zeigt Faktoren für eine erfolgreiche wirtschaftliche Entwicklung auf. Eine hinreichend große Zahl gut ausgebildeter Personen gepaart mit deren Bereitschaft zur Übernahme unternehmerischer Risiken sind zentrale Auslöser für eine sich selbst verstärkende wirtschaftliche Entwicklung. Diese hängt jedoch auch von einer Reihe weiterer Faktoren wie dem gleichzeitigen Vorliegen von Agglomerationseffekten, positiven externen Effekten (z.B. Wissens-Spillovers) und First-Mover-Effekten ab. Entscheidend ist, dass diese Effekte in ihrem Zusammenspiel nur schwer repliziert werden können. Dies ist zu bedenken, wenn die negativen Effekte einer unregulierten wirtschaftlichen Entwicklung reduziert werden sollen, was wiederum eine Überführung der Tätigkeiten in den formellen Sektor voraussetzt. Diese Überführung dürfte zwar ein entscheidender Faktor für die weitere Entwicklung des Sektors sein. Gleichzeitig kann hierdurch aber das schwer replizierbare Zusammenspiel der verschiedenen Erfolgsfaktoren empfindlich gestört werden. Sowohl Maßnahmen der nigerianischen Industriepolitik als auch Maßnahmen der deutschnigerianischen Zusammenarbeit in Wirtschafts- und Entwicklungsfragen erfordern daher eine umfassende Kenntnis über die Zusammenhänge zwischen diesen Erfolgsfaktoren.

Darüber hinaus ist zu diskutieren, unter welchen Umständen Nachteile wirtschaftspolitischer Eingriffe in Kauf genommen werden können, selbst wenn diese Nachteile geringer wiegen als die an anderer Stelle bewirkten Vorteile. So dürften Eingriffe zur Beseitigung von Marktversagen und zur Überführung der Tätigkeiten in den formellen Sektor regelmäßig begleitende Umverteilungsmaßnahmen erfordern, um eine Pareto-Verbesserung durch die Kompensation der Verlierenden aus den Gewinnen der Profitierenden der Maßnahmen zu erreichen. Vor dem Hintergrund von Informationsasymmetrien und Schwierigkeiten bei der Messbarkeit der Wohlfahrtswirkungen dieser Umverteilungsmaßnahmen, stellt deren Durchführung hohe Anforderungen an Regierungen und Behörden. 


\section{Literatur}

Abiola, A. B. (2008), The Otigba computer village cluster in Nigeria, in D. Z. Zeng (Hrsg.), Knowledge, technology, and cluster-based growth in Africa, 65-76, Washington, DC: The World Bank.

Asemota, V. (2017), Otigba: The experiment that grew into a tech market, https://guardian.ng/technology/otigba-the-experiment-that-grewinto-a-tech-market/ (21. Juli 2021).

Awosanya, Y. (2018), Relocation of Computer Village, Ikeja not happening earlier than 2020, https://techpoint.africa/2018/08/23/relocation-ofcomputer-village/ (21. Juli 2021).

BAMF (2021), Das Bundesamt in Zahlen 2020, Asyl.

Becker, B. und D. Goffart (2021), In Afrika geht es meinem Handy besser als hier, Wirtschaftswoche (20/2021).

BMZ (2021a), Das BMZ-Programm „Perspektive Heimat“.

BMZ (2021b), Strategische Partnerschaft Technologie in Afrika.

Carlino, G. und W. R. Kerr (2015), Agglomeration and Innovation, in G. Duranton et al. (Hrsg.) Handbook of Regional and Urban Economics, 5, 349-404.

Chen, S. (2019), Evolution of Settlement Pattern in Otigba Computer Village, Lagos State and its Challenges to Urban Planning, Advances in Multidisciplinary \& Scientific Research, 5(2).

Ekundayo, O. (2019), Education in Nigeria is in a mess from top to bottom. Five things can fix it, https://theconversation.com/education-in-nigeria-is-in-a-mess-from-top-to-bottom-five-things-can-fix-it-112894 (21. Juli 2021).

Famuyiwa, D. (2020), Traders stranded as Lagos state government demolishes popular Katangowa market, https://www.pulse.ng/news/local/ traders-stranded-as-lagos-govt-demolishes-popular-katangowamarket/p33mg01 (21. Juli 2021).

GIZ (2017), Digital Innovation Made in Africa for Sustainable and Inclusive Development.

GIZ (2020), Nigeria, https://www.giz.de/de/weltweit/1902.html (21. Juli 221).

GTAI (2020), Lohn- und Lohnnebenkosten I Nigeria, Arbeitsmarkt, Germany Trade \& Invest.

International Trade Administration (2020), Nigeria - Country Commercial Guide, Information and Communications Technology.

Jegede, O. (2019), A 'Computer Village' in Lagos offers lessons on what it takes to help small firms thrive. https://theconversation.com/acomputer-village-in-lagos-offers-lessons-on-what-it-takes-to-helpsmall-firms-thrive-118844 (21. Juli 2021).

National Bureau of Statistics (2021a), Labor Force Statistics: Unemployment and Underemployment Report (Q4 2020).

National Bureau of Statistics (2021b), Nigerian Gross Domestic Product Report (Q1 2021).

Nigerian Communications Commission (2021), Press Statement: Telecoms Sector, others Take Nigeria out of Recession as Sector Boosts GDP by $12.45 \%$.

OC\&C Consulting (2018), Tech entrepreneurship ecosystem in Nigeria.

Ogunjemilua, E. M., Oluwale, B. A., Jegede, O. O., und B.F. Ajao (2020), The nexus of knowledge sharing and innovations in the informal sector: The case of Otigba Hardware cluster in Nigeria, in International business and emerging economy firms, Palgrave Macmillan.

Omolayo, O. (2017), Ikeja Computer Village: What it was, what it will always be, https://techpoint.africa/2017/05/12/ikeja-computer-villagefeature/ (21. Juli 2021).

Onuoha, C. (2021), Relief comes to businessmen at Ikeja Computer Village, https://www.vanguardngr.com/2021/07/relief-comes-to-businessmen-at-ikeja-computer-village/ (21. Juli 2021).
Oyelaran-Oyeyinka, B. (2006), Learning in hi-tech and knowledge in local systems: The Otigba computer hardware cluster in Nigeria (Working Paper 2006-007), Institute for New Technologies, United Nations University.

Oyeyinka, O. (2017), Industrial clusters, institutions and poverty in Nigeria: The Otigba information and communications technology cluster, Springer.

Paha, J. und L. Wolter (2021), Otigba Computer Village - Erfolgsgeschichte basiert auf vielen Faktoren. Im Erscheinen: Entwicklung und Zusammenarbeit.

Päffgen, C. (2021), SWOT-Analyse | Nigeria, Entwicklung kommt nur schleppend voran, https://www.gtai.de/gtai-de/trade/wirtschaftsumfeld/swot-analyse/nigeria/entwicklung-kommt-nur-schleppend-voran-262630 (21. Juli 2021)

Porter, M. E. (1998), Clusters and the new economics of competition Harvard Business Review, 76(6), 77-90.

PwC (2016), Nigeria: Looking beyond Oil. PricewaterhouseCoopers.

Richardson, H.W. (1995), Economies and Diseconomies of Agglomeration, in Giersch H. (Hrsg.) Urban Agglomeration and Economic Growth, Springer.

Salaudeen, A. (2018), Welcome to Computer Village, Lagos, https://www. stearsng.com/article/welcome-to-computer-village-lagos (21. Juli 2021).

Scherer, K. (2018), Nigeria. Start-up Nation, 12. September, Zeit Online.

Sedgley, N., und B. Elmslie (2004), The Geographic Concentration of Knowledge: Scale, Agglomeration, and Congestion in Innovation Across U.S. States, International Regional Science Review, 27(2), 111-137.

Statistisches Bundesamt (2021), Außenhandel, Rangfolge der Handelspartner im Außenhandel der Bundesrepublik Deutschland.

Success Nwogu, I. (2018), Ikeja Computer Village generates N1.5bn daiIy -CAPDAN, https://punchng.com/ikeja-computer-village-generates-n1-5bn-daily-capdan/ (21. Juli 2021).

Technology Times (2014), Exclusive: Lagos State may need N20b for new Computer Village, https://technologytimes.ng/lagos-statemay-need-n20b-new-computer-village-photos/ (21. Juli 2021).

Udoma, U. U. und M. Belo-Osagie (2019), Nigeria: New National Minimum Wage Act, https://www.taylorvinters.com/article/nigeria-new-national-minimum-wage-act (21. Juli 2021).

Ulrich, A. (2017), Bildung in Nigeria, Der Schlüssel zum Glück, Deutschlandfunk Kultur.

Unesco (o. J.), Nigeria, Education and Literacy, United Nations Educational, Scientific and Cultural Organization.

Unicef (o. J.), Nigeria. Education, United Nations International Children's Emergency Fund.

United Nations, Department of Economic and Social Affairs, Population Division (2019a), World Population Prospects 2019, Volume I.

United Nations, Department of Economic and Social Affairs, Population Division (2019b), World Population Prospects 2019, Volume II.

Vanguard News Nigeria (2020a), LASG reassures Computer Village stakeholders of seamless relocation to Katangua, https://www.vanguardngr.com/2020/03/lasg-reassures-computer-village-stakeholdersof-seamless-relocation-to-katangua/ (21. Juli 2021).

Vanguard News Nigeria (2020b), Relocation: New 'Computer Village' ready in 24 months - Developer, https://www.vanguardngr.com/2020/09/ relocation-new-computer-village-ready-in-24-months-developer/ (21. Juli 2021).

Vanguard News Nigeria (2021), Lagos reiterates commitment to relocate Computer Village, https://www.vanguardngr.com/2021/05/lagosreiterates-commitment-to-relocate-computer-village/ (29. Juli 2021). Weltbank (2020), World Bank National Accounts Data.

Weltbank (2021), Poverty \& Equity Brief, Africa Western \& Central, Nigeria.

Title: IT Sector: Opportunities for the German-Nigerian Economic and Development Partnership

Abstract: The emergence and growth of the largest African market for information and communication technology in Lagos, Nigeria, is based on agglomeration effects and the interaction between complementary success factors that have positive externalities. Necessary reforms that are supposed to be implemented through national measures to eliminate market failures as well as international measures in development policy require a market transition into the formal economy. However, this necessity runs the risk of destroying the successful interplay between the factors of success. This article illustrates the opportunities and risks for German-Nigerian economic and development cooperation. 